\title{
Combined Scanning Probe Microscopy and Confocal Raman Spectroscopy for Functional Imaging of the Layered Materials
}

Anton V. Ievlev ${ }^{1,2}$, Michael A. Susner ${ }^{3}$, Michael A. McGuire ${ }^{3}$, Petro Maksymovych ${ }^{1,2}$ and Sergei V. Kalinin ${ }^{1,2}$

${ }^{1 .}$ Center for Nanophase Materials Sciences, Oak Ridge National Laboratory, Oak Ridge, TN

2. Institute for Functional Imaging of Materials, Oak Ridge National Laboratory, Oak Ridge, TN

3. Materials Science and Technology Division, Oak Ridge National Laboratory, Oak Ridge, TN

Comprehensive investigations of the nano-structured materials and devices are impossible without development of the modern investigative techniques, allowing noninvasive imaging of the processes defining materials functionality on the nanoscale. Scanning probe microscopy (SPM) is one of such paradigmatic tools. Since its development, about thirty years ago, it has given rise to numerous ancillary techniques from basic topography mapping to complex investigations of the ionic and electronic motion, as well as coupled electrochemical and electromechanical functional response in almost all possible environmental conditions [1-3]. However, the capabilities of SPM are limited by the properties of the tip-surface junction, which introduces a number of shortcomings, including limited time resolution, reduced to null sensitivity to bulk response and elusive chemical and crystallographic information. Introduction of other, concomitant microscopic and spectroscopic techniques into the measurement setup can not only provide a solution to these shortcomings, by enhancing resolution, but also augment the overall information obtained, with infinite opportunities for comprehensive nanoscale studies of the coupling between different material properties (electrical, mechanical, optical, chemical, etc.).

Here we used approach combining SPM and confocal Raman spectroscopy for nanoscale functional study of copper indium thiophosphate $\left(\mathrm{Cu}_{1-\mathrm{x}} \mathrm{In}_{1+\mathrm{x} / 3} \mathrm{P}_{2} \mathrm{~S}_{6}\right)$ layered ferroelectric [4]. Despite significant attention to investigations of this material during last decade, nanoscale origin of its electronic properties remains poorly discovered.

Performed investigations allowed to study in details ferroelectric-paraelectric phase transition. The insitu measurements by Piezoresponse force microscopy (PFM) [3] and Raman spectroscopy revealed correlated changes in the ferroelectric domain structure and Raman spectra during laser heating. However, non-polar inclusions of $\operatorname{In}_{4 / 3} \mathrm{P}_{2} \mathrm{~S}_{6}$ hampered direct identification of the phase transformation. The problem was resolved using Bayesian linear unmixing and principal component analysis for the separation of the Raman spectra corresponding to different material phases. These results, along with the macroscopically measured Curie temperatures and finite element simulation of the temperature distribution, allowed us to calibrate the temperature in the irradiated region close to the tip.

Results of the current research are important for fundamental studies of the dielectric layered materials and development of novel SPM techniques for systematic studying of temperature dependent material functionalities in previously inaccessible temperature regimes [5].

\section{References:}

[1] Binning G.K, Phys. Scr., T19A (1987), p.53

[2] Schönenberger C., Alvarado, S. F., Phys. Rev. Lett., 65, (1990), p.3162. 
[3] Kalinin S.V., et.al. , Rep. Prog Phys, 73 (2010), p. 056502.

[4] Ievlev, A.V., et.al, ACS Nano, 9(12), (2015), p. 12442.

[5] Research supported by Oak Ridge National Laboratory's Center for Nanophase Materials Sciences

(CNMS), which is a U.S. Department of Energy, Office of Science User Facility.
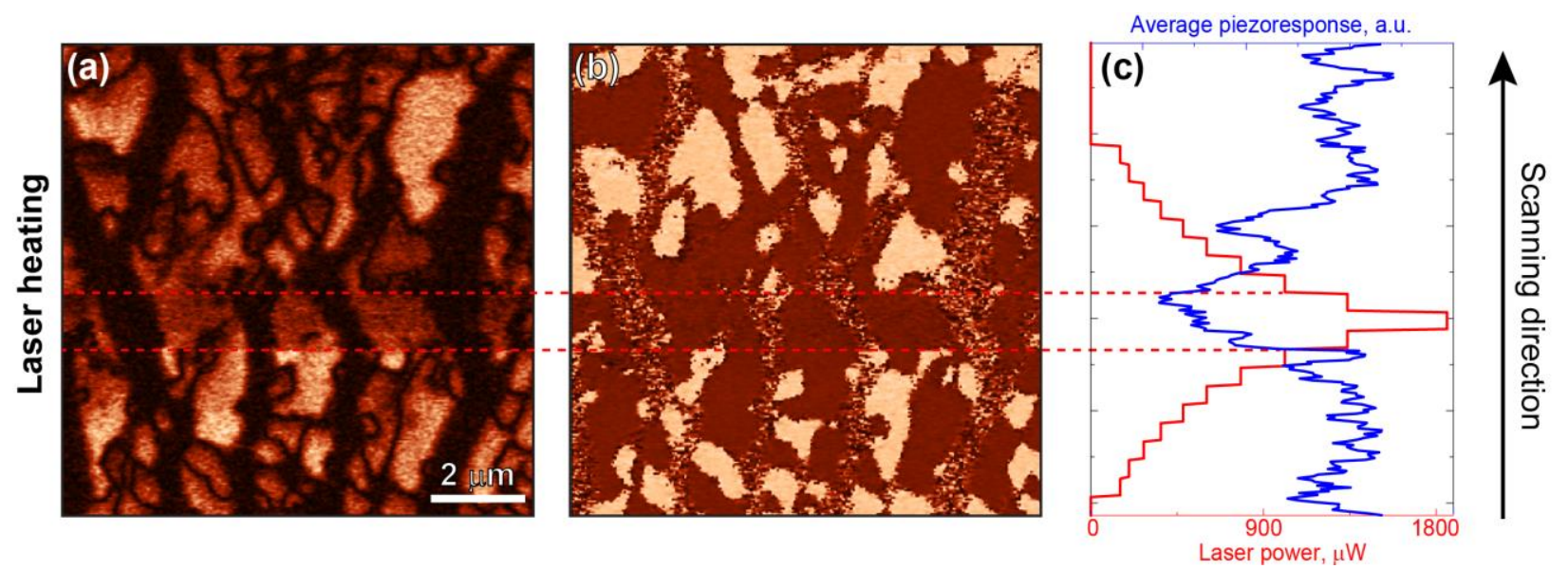

Figure 1. Changes of the ferroelectric domain structure induced by local laser irradiation. (a) PFM measurements during heating with various laser intensities; we see the piezoresponse amplitude in (a) and the piezoresponse phase in (b). (c) Plot of laser power and average amplitude of the piezoresponse in ferroelectric CuInP2S6 and paraelectric $\operatorname{In}_{4 / 3} \mathrm{P}_{2} \mathrm{~S}_{6}$ phases.

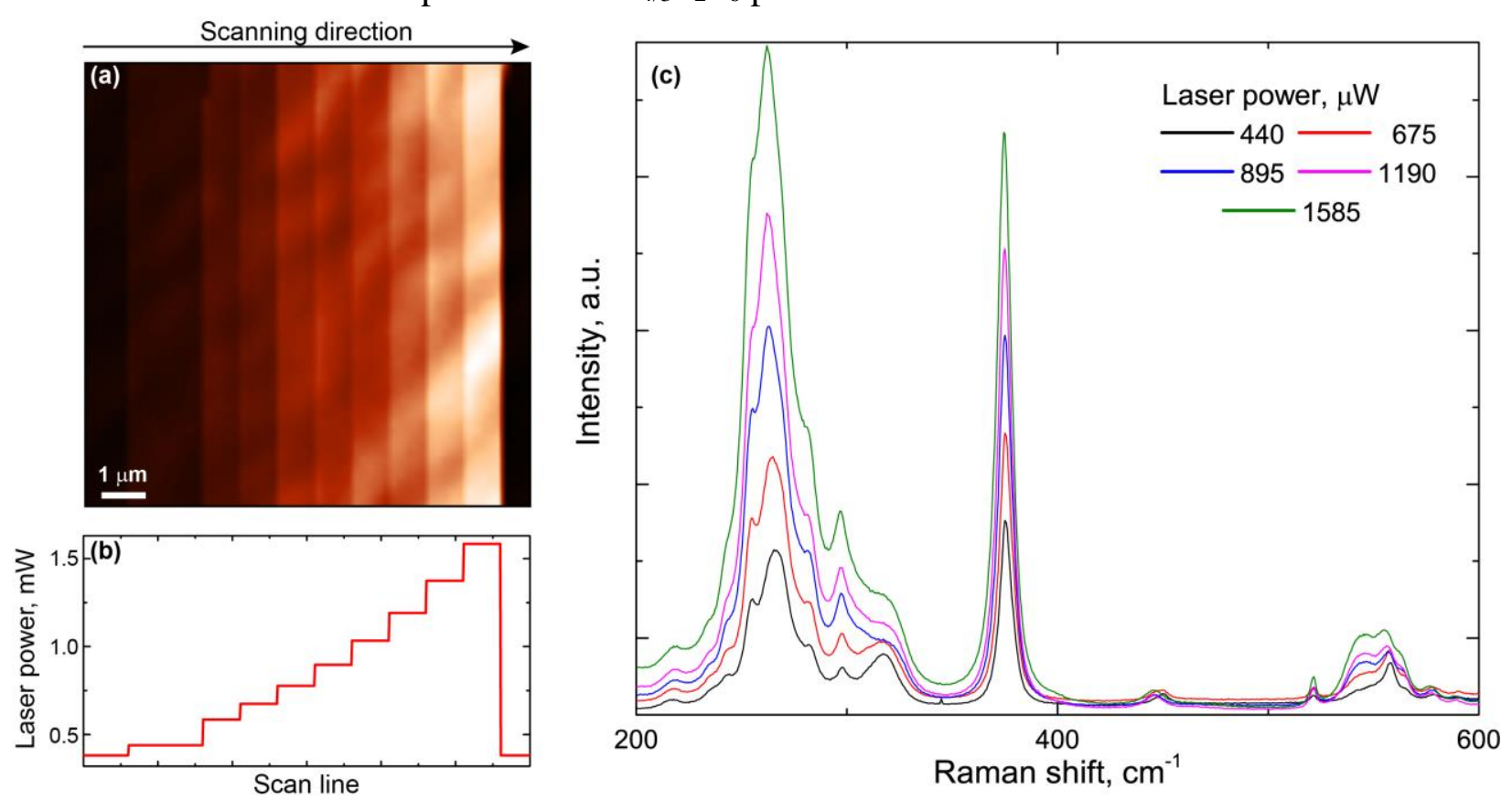

Figure 2. Raman spectra mapping with different laser intensities. (a) Map of the integral Raman intensity. (b) Plot of the laser power. (c) Raman spectra averaged over regions with fixed irradiation power. 